\title{
Embryonic Synthesis of the Inner Limiting Membrane and Vitreous Body
}

\author{
Willi Halfter, ${ }^{1}$ Sucai Dong, ${ }^{1}$ Barbara Schurer, ${ }^{1}$ Colleen Ring, ${ }^{2}$ Gregory J. Cole, ${ }^{3}$ and \\ Andrew Eller ${ }^{4}$
}

Purpose. The inner limiting membrane (ILM) and the vitreous body (VB) are major parts of the extracellular matrix of the eye. The present study was undertaken to investigate the synthesis and turnover of the ILM and VB in chick and human embryonic and postembryonic eye development.

Methods. The abundance of ILM and VB proteins was determined by Western blot analysis using samples from chick and human VB of different ages. The mRNA expression of the ILM proteins in lens was determined by in situ hybridization and RT-PCR.

RESUlts. Based on the abundance of mRNA expression, the prominent sources of ILM and VB proteins in chick eyes are the lens and ciliary body. In chick, ILM and VB matrix proteins were most abundant in embryonic $\mathrm{VB}$, and their concentration declined precipitously after hatching. Most ILM and VB proteins were no longer detectable in the adult VB. In humans, a similar developmentally regulated expression of ILM and VB proteins in VB was detected: The highest concentrations of ILM and VB proteins were detected in fetal VB, the lowest in the adult VB. The decline in ILM and VB protein synthesis occurred within the first 2 years of life.

Conclusions. The abundance of ILM and VB proteins in the embryonic VB, their sharp decline at postembryonic stages, and their very low abundance in the adult VB show that ILM and VB are assembled during embryogenesis and are maintained throughout life with minimum turnover. (Invest Ophthalmol Vis Sci. 2005;46:2202-2209) DOI:10.1167/iovs.041419

B asement membranes (BMs) are sheets of extracellular matrix (ECM) that separate epithelia, myofibers, blood vessels, and the nervous system from the adjacent interstitial connective tissues. ${ }^{1}$ Major components of BMs include members of the laminin family, collagen IV, nidogen, perlecan, agrin, and collagen XVIII. ${ }^{2,3}$ The inner limiting membrane (ILM) is the BM of the retina and separates the retina from the vitreous body (VB). Ganglion cell death and extensive retinal dysplasia after embryonic ILM disruption in chicks ${ }^{4,5}$ and $\mathrm{mu}-$ tant mice ${ }^{6,7}$ showed that the ILM is necessary for normal retinal

From the Departments of ${ }^{1}$ Neurobiology and ${ }^{4}$ Ophthalmology, University of Pittsburgh, Pittsburgh, Pennsylvania; the ${ }^{2}$ Department of Neuroscience, University of North Carolina, Chapel Hill, North Carolina; and the ${ }^{3}$ Department of Molecular Biological Sciences, North Carolina State University, Raleigh, North Carolina.

Supported by National Science Foundation Grant IBN-0240774.

Submitted for publication December 4, 2004; revised January 22 , 2005; accepted March 4, 2005.

Disclosure: W. Halfter, None; S. Dong, None; B. Schurer, None; C. Ring, None; G.J. Cole, None; A. Eller, None

The publication costs of this article were defrayed in part by page charge payment. This article must therefore be marked "advertisement" in accordance with 18 U.S.C. $\$ 1734$ solely to indicate this fact.

Corresponding author: Willi Halfter, Department of Neurobiology, University of Pittsburgh, 1402 East Biological Science Tower, Pittsburgh, PA 15261; whalfter@pitt.edu. development. Further, eyes affected by a variety of congenital human diseases, summarized as type II lissencephaly, show retinal dysplasia combined with disruptions in the ILM. ${ }^{8-10}$ In all instances, the histogenesis of other parts of the central nervous system is altered as well, most dramatically that of the cerebral cortex. ${ }^{11-14}$

The largest ECM compartment of the eye is the VB. The gel-like structure consists of $98 \%$ water. Collagens II and IX, fibronectin, fibrillin, and opticin are the most prominent ECM constituents (reviewed in Refs. 15-17). A major component of the mammalian VB is hyaluronic acid, ${ }^{18}$ which is replaced in the chick by a highly glycosylated version of collagen IX. ${ }^{19}$ The gel structure of the VB is dependent on its collagen constituents and not on the presence of hyaluronic acid. ${ }^{20}$ In situ hybridization in mice and chick localized the site of synthesis of VB proteins to the ciliary body (reviewed in Refs. 16,17, 21).

In the present study, we investigated the assembly and turnover of the ILM and VB during development. A surprising finding was that ILM and VB were synthesized during embryogenesis and maintained from early postnatal life onward, with very low turnover.

\section{Methods}

\section{Antibodies}

The antibodies to chick laminin-1, nidogen-1, perlecan, collagen XVIII, agrin, and collagen IX have been described previously. ${ }^{2,23}$ The antibodies are mouse monoclonals (mAbs) and are available from the Developmental Studies Hybridoma Bank, University of Iowa (Iowa City, IA). The specificities of mAbs $3 \mathrm{~F} 1$ to collagen II, $2 \mathrm{~A} 6$ to fibrillin, $5 \mathrm{~F} 3$ to fibronectin, and $11 \mathrm{~F} 9$ to chick albumin were established by immunocytochemistry (collagen II, fibrillin, and fibronectin), Western blot analysis (collagen II, fibrillin, and fibronectin), cross-reactivity of the immunoprecipitated antigens with polyclonal antisera (collagen II, fibrillin, and fibronectin), or cDNA cloning from a chick amnion phage cDNA library using the antibody as a probe (albumin). The mAbs to tenascin (mAb M1), human photoreceptors (RC2), and synaptic vesicles (SV2) were obtained from the Developmental Studies Hybridoma Bank. Polyclonal antisera to collagens II and IV, laminin-1, and transferrin were obtained from Rockland Immunochemicals (Gilbertsville, PA), Chemicon (Temecula, CA), Invitrogen (Carlsbad, CA), and Cappel (West Chester, PA), respectively. The polyclonal antiserum raised against chick collagen XVIII-endostatin will be described elsewhere (Dong S, Winzen U, Sasaki T, Timpl R, Halfter W. Endostain is necessary for collagen XVIII triple helix formation, manuscript submitted, 2005). The polyclonal antisera were used to detect ILM and VB proteins in human eyes and collagen IV in chick eyes.

\section{Western Blot Analysis}

VBs were collected from embryonic day (E)10 and E20, postnatal day (P)5 and P10, and adult chickens. Adult chicken eyes were obtained from Greenwalt Farms (Madison, PA). The VB samples were collected within less than hour of killing and were the combined VBs of at least three different animals. VBs of fetal and early postnatal human eyes were obtained from the Brain and Tissue Bank of the University of 
Maryland (Baltimore, MD). For the fetal sample, three VBs were pooled from three 19-week-old fetuses; the postnatal samples were each of 96and a 253-day-old infant who had died of pneumonia and cardiomyopathy, respectively. The VBs of two adult eyes were from the Center for Organ Recovery and Education, Pittsburgh, PA (IRB protocol 0312072). The eyes were obtained and managed in accordance with the guidelines of the Declaration of Helsinki for research involving human tissue. After homogenization and centrifugation, the samples were run on SDS-PAGE ${ }^{23}$ and the Western blots probed for laminin-1, collagen IV, perlecan, collagen XVIII, and agrin. The relative abundance of the proteins at the different time points was quantified by measuring the total gray value of the protein bands from the digital images on computer (NIH Image software 1.63; Scion, Frederick, MD). The total gray value was calculated by multiplying the pixel number by the optic density of the bands and plotted as multiples of the gray values of equally sized, nonlabeled segments of the blots. Liquefaction of the chick VB was determined at different embryonic and posthatching stages by weighing the liquid and gel portions of the VB.

\section{In Situ Hybridization}

For in situ hybridization, eyes from E4 to E10 chicks were fixed overnight in $4 \%$ paraformaldehyde. Cryostat sections were processed for in situ hybridization using the digoxigenin (DIG)-labeled cRNA probes to chick perlecan, collagen XVIII, agrin, fibronectin, and collagen IX as described previously. ${ }^{24}$

\section{RT-PCR of ILM Proteins}

For the detection of mRNA levels for perlecan, nidogen, and collagen IV by RT-PCR, lenses from embryonic and posthatching chicks were dissected, their total RNA extracted (Mico-to-Midi Total RNA Isolation System; Invitrogen) and 200- to 1200-bp sequences of perlecan, nidogen, and collagen IV amplified by RT-PCR (One-Step RT-PCR kit; Invitrogen). The samples were normalized by using RT-PCR for chick $\beta$-actin as the control. The primers are perlecan forward, 5' ACC ATC AAG GAT GCC TAC AAG A; reverse, 5'CGC TCA CTG CCT CCC CTT CA; nidogen forward, 5'CAG CCA ATG ACT GTT TTG TAG CA; reverse, 5'TGA CAG AAG TGG GCT TGC ATG T; $\beta$-actin forward, 5'CAT GAA ACT ACC TTC AAC TCC A; reverse, 5'TAG AAG CAT TTG CGG TGG ACA A; collagen IV forward, 5'ATG GCT ACT CCC TTC TTT ATG TA; reverse, 5'TTT CTT GAA CAT CTC ATT CCT CTC.
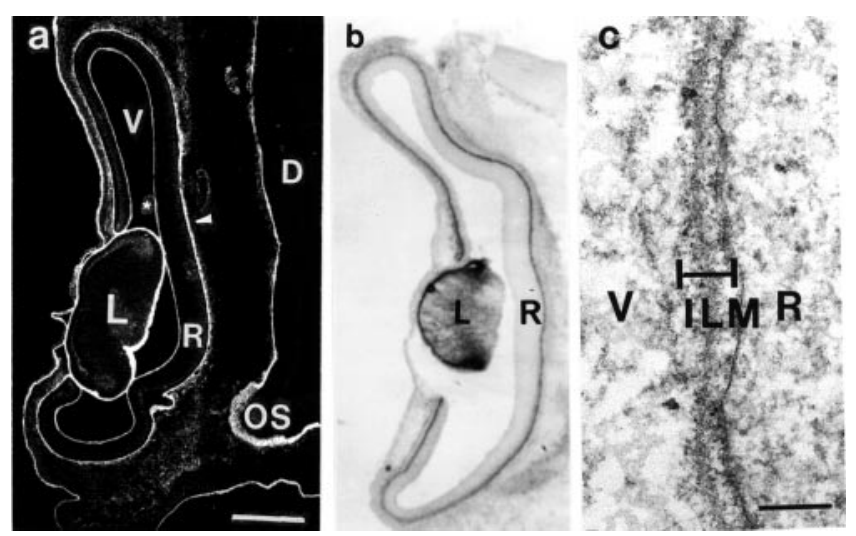

FigurE 1. BMs of the E4 chick eye visualized by immunostaining for perlecan (a). The lens capsule (L), the ILM ( $\star$ ), and Bruch's membrane (arrowbead) were strongly labeled in the cross section. The antibody also labeled the pial BM of the diencephalon (D) and BM outlining the optic stalk (OS). Perlecan mRNA was expressed in the lens but not the retina, as shown by in situ hybridization (b). High-power electron microscopy (c) shows the ILM as a 80-nm thick sheet of ECM between the retina (R) and the ECM of the VB (V). Bars: (a, b) $200 \mu \mathrm{m}$; (c) $100 \mathrm{~nm}$.

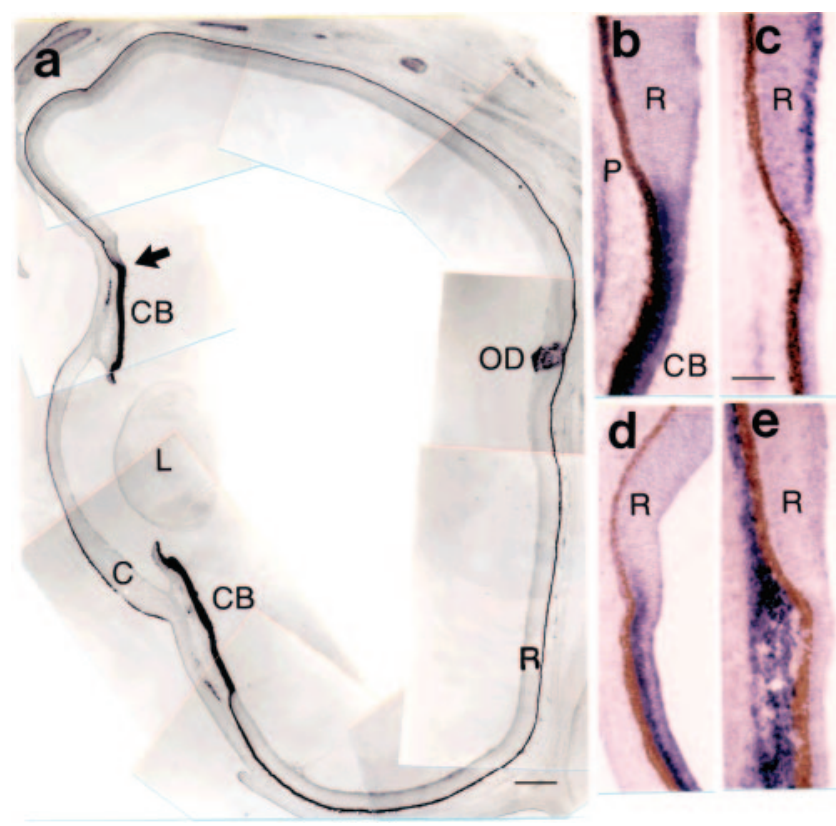

Figure 2. Cross sections of E10 chick eyes showing the sites of mRNA expression of collagens XVIII and IX, agrin, and fibronectin by in situ hybridization. Collagen XVIII and agrin are ILM proteins, and collagen IX and fibronectin are prominent components of the VB. A low-power view of an entire E10 eye (a) shows the expression of collagen XVIII mRNA in the ciliary body (CB). High-power views of the ciliary segment (arrow) show that the nonpigmented ciliary epithelium is the site of synthesis of collagens XVIII (b) and IX (d). Exceptions are agrin and fibronectin: agrin is synthesized by retinal ganglion cells (c) and fibronectin by extraocular connective tissue (e). C, cornea; OD, optic disk; R, retina. Bars: (a) $200 \mu \mathrm{m}$; (b-e) $75 \mu \mathrm{m}$.

RT-PCR conditions were at room temperature: 1 cycle $50^{\circ} \mathrm{C} 30$ minutes and $94^{\circ} \mathrm{C}$ for 2 minutes; PCR, 35 cycles: $94^{\circ} \mathrm{C} 15$ seconds, $56^{\circ} \mathrm{C} 30$ seconds, $72^{\circ} \mathrm{C} 1$ minute 10 seconds; 1 cycle for final extension: $72^{\circ} \mathrm{C}$ for 5 minutes. PCR products were separated on $1 \%$ agarose gels.

\section{Results}

\section{ILM and VB Protein mRNA Expression in the Developing Chick Eye}

The ILM of the chick retina is an 80-nm thick sheet of ECM that is located at the vitreal surface of the retina and blends into the ECM of the VB (Figs. 1a, 1c). The ILM is composed of perlecan, collagen IV, laminin-1, nidogen, agrin, and collagen XVIII, as confirmed by immunocytochemistry (Fig. 1a) and Western blot analysis. ${ }^{23}$ In situ hybridization showed that ILM protein $\mathrm{mR}$ NAs in the chick eye are prominently expressed by the lens, ciliary body, and optic disc. Except for agrin, ILM protein mRNAs were not detected in the retina. Perlecan mRNA, for example, was most abundant in the lens (Fig. 1b), whereas collagen XVIII mRNA was most abundant in the nonpigmented epithelium of the ciliary body and the optic disc (Figs. 2a, 2b). Similar distribution patterns were established for laminin, nidogen, and collagen IV mRNAs. ${ }^{23,24}$ The only ILM protein mRNA that was detectable in the retina was that for agrin, which was prominently expressed by ganglion cells (Fig. 2c).

VB protein mRNAs were detected in the nonpigmented ciliary body epithelium, as shown for collagen IX (Fig. 2d). Similar distribution patterns were established for collagen $\mathrm{II}^{25}$ and tenascin. ${ }^{26}$ The only ECM protein in VB that does not originate from the ciliary body is fibronectin. Fibronectin 


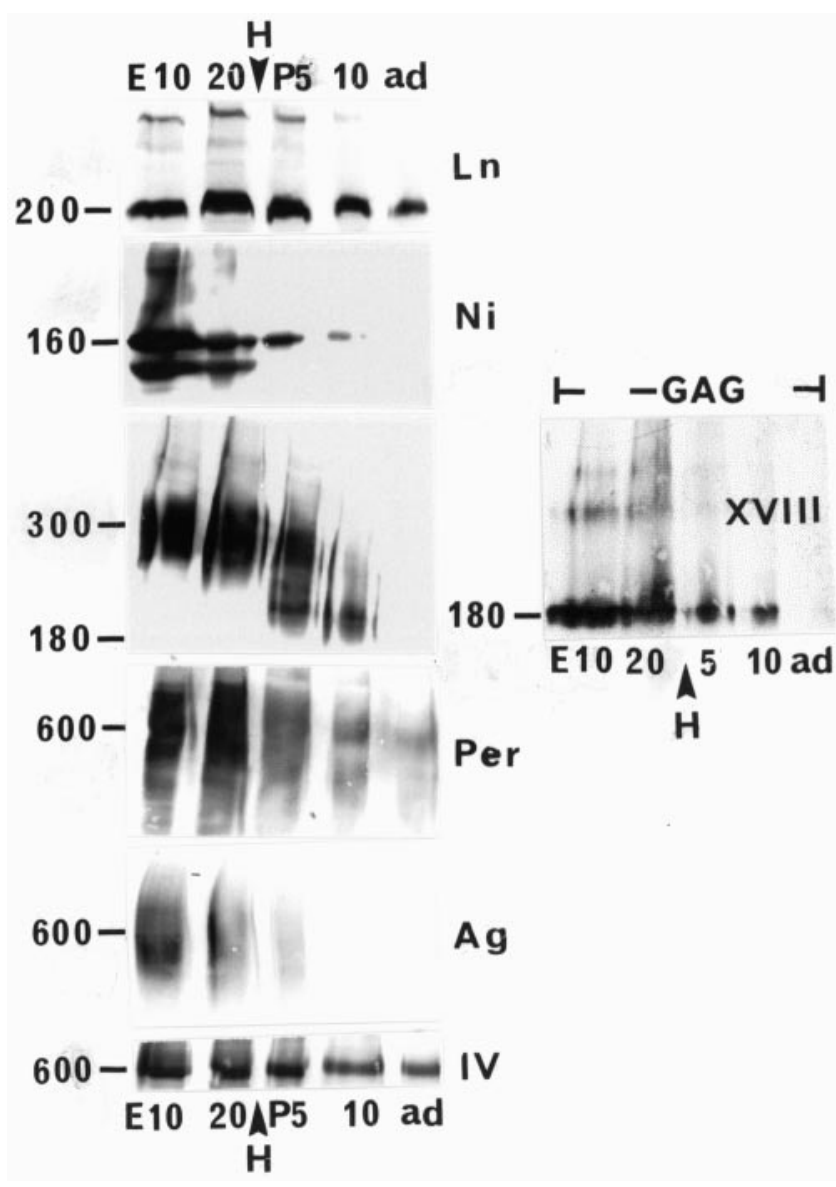

Figure 3. Western blot analysis showing the abundance of laminin-1 (Ln), nidogen 1 (Ni), collagen XVIII (XVIII), perlecan (Per), agrin (Ag), and collagen IV (IV) in E10, E20, (P)5, P10, and adult (ad) chicken VB. $\mathrm{H}$ : hatching at E21. In each lane, $40 \mu \mathrm{L}$ of VB homogenate was loaded. All proteins declined in concentration shortly after hatching. The decrease in molecular mass of collagen XVIII was due to an age-related glycosylation deficiency rather than to degradation of the core protein. When the samples were separated after treatment with heparitinase (-GAG; right, XVIII), the molecular mass of the $180-\mathrm{kDa}$ core protein remained unchanged.

mRNA was undetectable in any of the ocular tissues and was found only in extraocular connective tissue (Fig. 2e). Consistent with earlier studies in chicks ${ }^{24}$ and mice, ${ }^{27,28}$ we propose that ILM and VB proteins in chicks originate from the lens and ciliary body, although a contribution of retinal glial cells in ILM synthesis cannot be excluded.

\section{Developmental Regulation of ILM Synthesis in Chicks}

Based on abundant mRNA expression, the dominant source for ILM proteins in the chick eye are the lens, ciliary body, and optic disc. We postulate that for the assembly of the ILM from its protein constituents, the $\mathrm{BM}$ proteins have to be secreted from the lens and ciliary body into the $\mathrm{VB}$, and a requirement for the assembly of new ILM or for the turnover of alreadyexisting ILM is the availability of ILM proteins in the VB. Western blot analysis confirmed that all ILM proteins were abundant in the embryonic $\mathrm{VB}$, at a time when the retina rapidly expands, and large segments of new ILM are assembled (Fig. 3). ${ }^{23}$ The ILM protein concentrations in VB sharply declined during the first 10 days after hatching, and most proteins were no longer detectable in the adult VB (Fig. 3). Particularly striking was the dramatic downregulation of nidogen that was most abundant in E10 vitreous, less abundant by E20, barely detectable by P10, and no longer detectable in the adult VB (Fig. 3, Ni). Collagen XVIII, a heparan sulfate proteoglycan, ${ }^{29}$ was detected by E10 and E20 as a smear of $300 \mathrm{kDa}$ (Fig. 3, XVIII). The staining intensity of the smear was weaker by $\mathrm{P} 5$ and the collagen XVIII band decreased in molecular mass. The decline in molecular mass was even more pronounced by P10. To determine whether the decrease was due to a proteolytic degradation of the core protein, the samples were digested with heparitinase to remove the GAG chains (Fig. 3, - GAG). Western blot analysis showed that the $180-\mathrm{kDa}$ collagen XVIII core protein remained unchanged during all stages of development, showing that the decrease in molecular mass of the collagen XVIII proteoglycan reflects an insufficient postembryonic GAG-glycosylation or a progressive deglycosylation, rather than a proteolysis of the core protein.

Quantification of the Western blots by scanning densitometry showed that the expression of ILM proteins was most abundant during embryogenesis (Figs. 3, 4). Nidogen, collagen XVIII, and agrin concentrations dramatically declined shortly after hatching, and the three proteins were no longer detectable in the adult chick eye. Laminin-1, perlecan, and collagen IV concentrations also declined after hatching, but the proteins were still detectable, although at lower levels, in the adult eye (Figs. 3, 4).

\section{mRNA Expression of ILM Proteins at Different Stages of Development}

The relative mRNA levels of perlecan, nidogen, and collagen IV were determined by RT-PCR at different stages of eye development (Fig. 5). The mRNA levels for collagen IV, nidogen, and perlecan were highest at E8 and declined at E14, at a time when the proteins were still abundant in VB (Fig. 5, compare
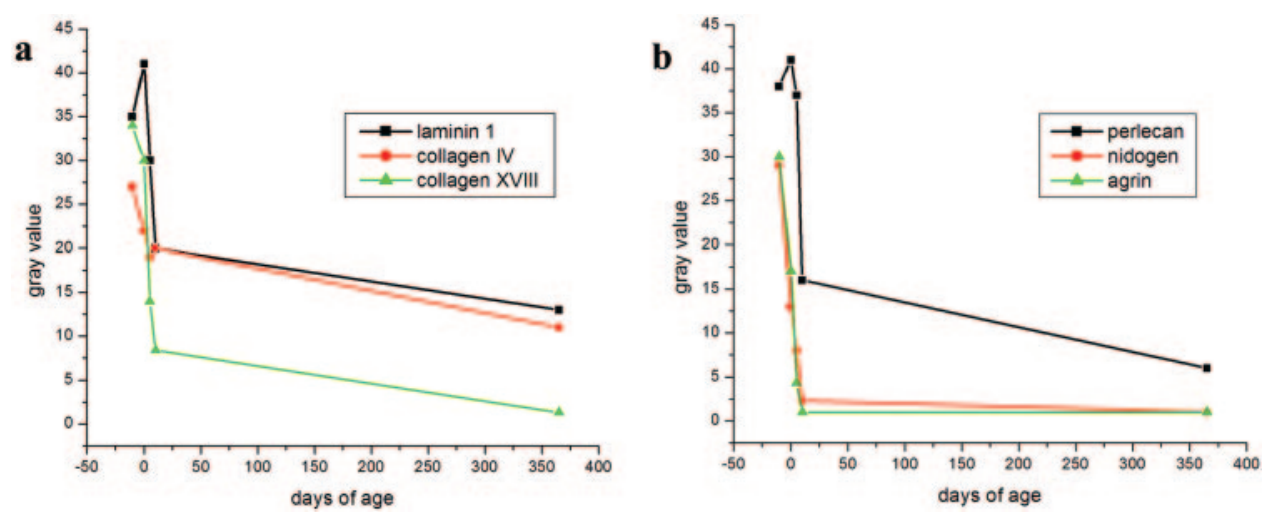

Figure 4. (a, b) Quantification of abundance of the ILM proteins (shown in the keys) in the embryonic and posthatching chick VB. Gray values of the proteins were determined by scanning densitometry and plotted against age. The adult eyes were from 1-year-old chickens. 
$\begin{array}{llllllllllll}\text { St } & \text { E8 } & E 14 & P 10 & A D & \text { E8 } & \text { E14 } & \text { P10 } & A D\end{array}$
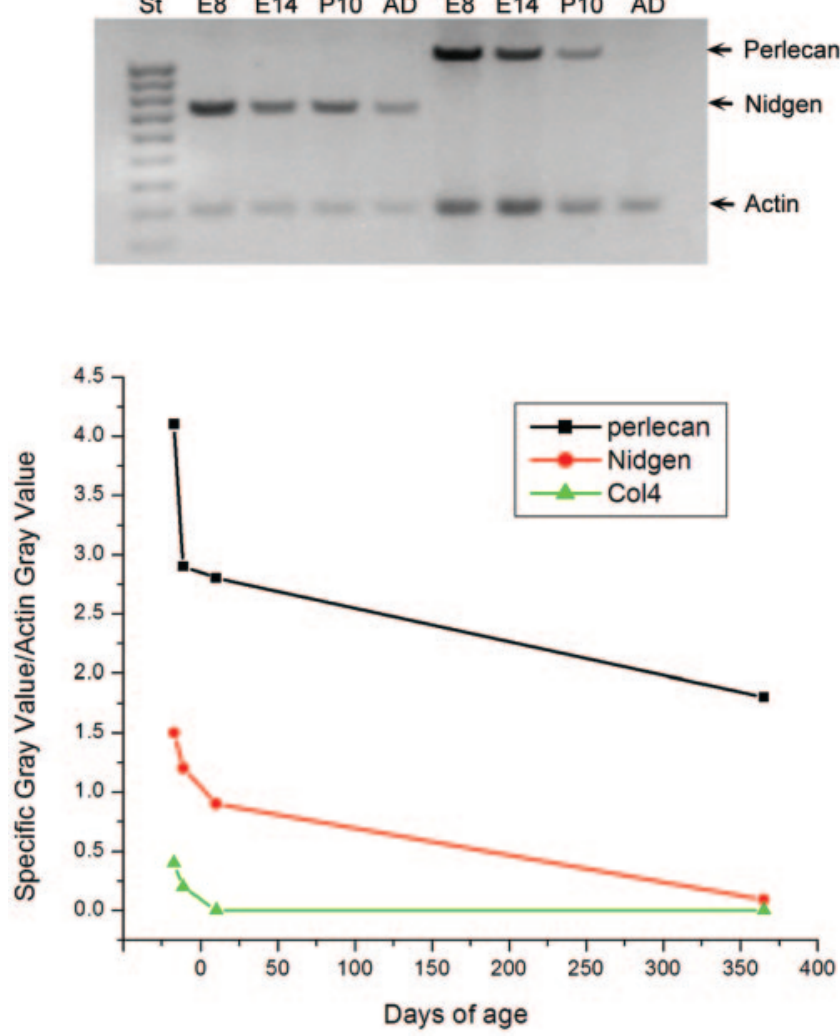

FIGURE 5. mRNA expression of perlecan, nidogen, and $\beta$-actin in lens at different stages of pre- and posthatching development, by RT-PCR. Both perlecan and nidogen mRNAs were highest at E8 and were downregulated from E14 onward. Densitometry of amplified nidogen, perlecan, and collagen IV bands after normalization against actin showed that the mRNAs for perlecan, nidogen, and collagen IV were highest in the early embryonic stage and decreased in concentration at late embryonic stages, well proceeding the protein decline in the VB.

with Figs. 3, 4). Very little nidogen mRNA was detectable in the adult chick eye.

mRNA levels for ILM proteins synthesized by the ciliary body, such as collagen XVIII, laminin- $\beta 1,-\gamma 1$, and $-\alpha 1$ could not be measured, because the chick ciliary body epithelium was inseparable from the periocular connective tissue and could not be isolated as a defined, clean tissue.

\section{Developmental Regulation of VB Body Proteins in the Chick}

Western blot analysis of VB samples from embryonic and posthatching chickens showed that the abundance of VB proteins was highest during embryonic stages. It rapidly declined during the first 10 days after hatching, and little collagen IX, fibrillin, and tenascin and only minor quantities of collagen II were detectable in adult VB (Fig. 6a). An exception was fibronectin, which was present in similar quantities from embryonic stages of development into adulthood. The embryonic $\mathrm{VB}$ was also rich in proteins that are typical of serum, such as albumin and transferrin. Whereas albumin was greatly reduced in concentration shortly after hatching and was undetectable in adult $\mathrm{VB}$, transferrin was detectable throughout all stages of chick eye development into adulthood (Fig. 6).

Quantification of the protein bands showed a similar downregulation of VB proteins as was shown in the ILM components (Figs. 6b, 6c). The highest concentration of VB proteins was detected at embryonic stages, followed by a rapid decline during the first 10 days after hatching. Only low levels of the proteins were detectable in the adult VB. The evenly high concentrations of fibronectin and transferrin showed that the decline of the other VB proteins was not due to an increased and random proteolysis in the posthatching VB. In situ hybridization showed that fibronectin is not synthesized by the eye, rather from extraocular tissue (Fig. 2e). The origin of transferrin in chick VB has not been determined, yet in rabbit, transferrin has been shown to be secreted by the ciliary epithelium. ${ }^{30}$

Age-related liquefaction, as documented in humans (reviewed in Refs. 15-17), is considered a morphologic sign of VB degradation. To find out whether VB liquefaction occurs in chicks as well, the proportion of liquid versus total VB weight was determined (Fig. 7). Data showed that $20 \%$ of the embryonic VB was liquid. That fraction increased to $44 \%$ at P10 and to $75 \%$ in adult chicks, demonstrating that liquefaction in chicks begins as soon as the early posthatching stages of eye development.

\section{Developmental Expression of ILM and VB Proteins in the Human Eye}

The expression levels of ILM and VB proteins during human development were investigated as well. We first confirmed the presence of all known ILM proteins, such as collagen IV (Fig. 8d), laminin (Fig. 8e), nidogen (Fig. 8f), perlecan (Fig. 8g), collagen XVIII (Fig. 8h), and agrin in the adult human ILM (Fig. $8 i)$. For orientation, sections were stained with a general nuclear marker (Fig. 8a), for RC2 as a marker of photoreceptors (Fig. 8b), and for SV2 as a marker for the outer and inner plexiform layers (Fig. 8c). Western blot analysis confirmed the specificity of the anti-ILM antibodies as they labeled protein bands with the expected molecular masses in samples of VB (Fig. 8k).

When assayed for different stages of pre- and postnatal eye development, laminin-1 (Fig. 8k, LN), collagens XVIII (Fig. 8k, XVIII), IV (Fig. 8k, IV), and II (Fig. 8k, II) were most abundant in 19-week-old fetal VB (Fig. 8k, lane 1). By 2.4 months after birth, less ILM and VB proteins were detected in VB (Fig. 8k, lane 2), and the proteins decreased further in concentration by 6.3 months of age (Fig. 8k, lane 3). The proteins were barely detectable in the VB of adult eyes (Fig. 8k, lane 4). Quantification of the blots (Fig. 81) showed a protein expression profile that was very similar to the temporal expression of ILM and VB proteins in chick: ILM and VB proteins were most abundant at fetal stages, less in early postnatal stages, even less in slightly older stages, and least in the adult eye. There was a dramatic decline in ILM and VB protein abundance shortly after birth.

\section{Discussion}

In the present study, we investigated the metabolism of the ILM and VB during chick and human eye development. Western blot analysis and RT-PCR showed that the synthesis and assembly of the ILM and VB in chicks and humans occurs during embryonic development and is dramatically reduced in postembryonic life. The downregulation of ILM and VB synthesis in humans occurs within the first few years after birth.

\section{Embryonic Synthesis of the ILM}

Consistent with previous reports, ${ }^{23,24}$ the mRNA expression of most ILM proteins in the chick eye was localized to the lens, ciliary body, and optic disc. Similar studies in mice have localized the mRNA expression for laminin, nidogen, and collagen IV to the ciliary body and lens as well. ${ }^{27,28}$ We assume a similar mRNA expression pattern for the human eye. Based on mRNA expression, we postulate that ILM proteins are mainly synthesized by the lens and ciliary body. The absence of ILM protein 

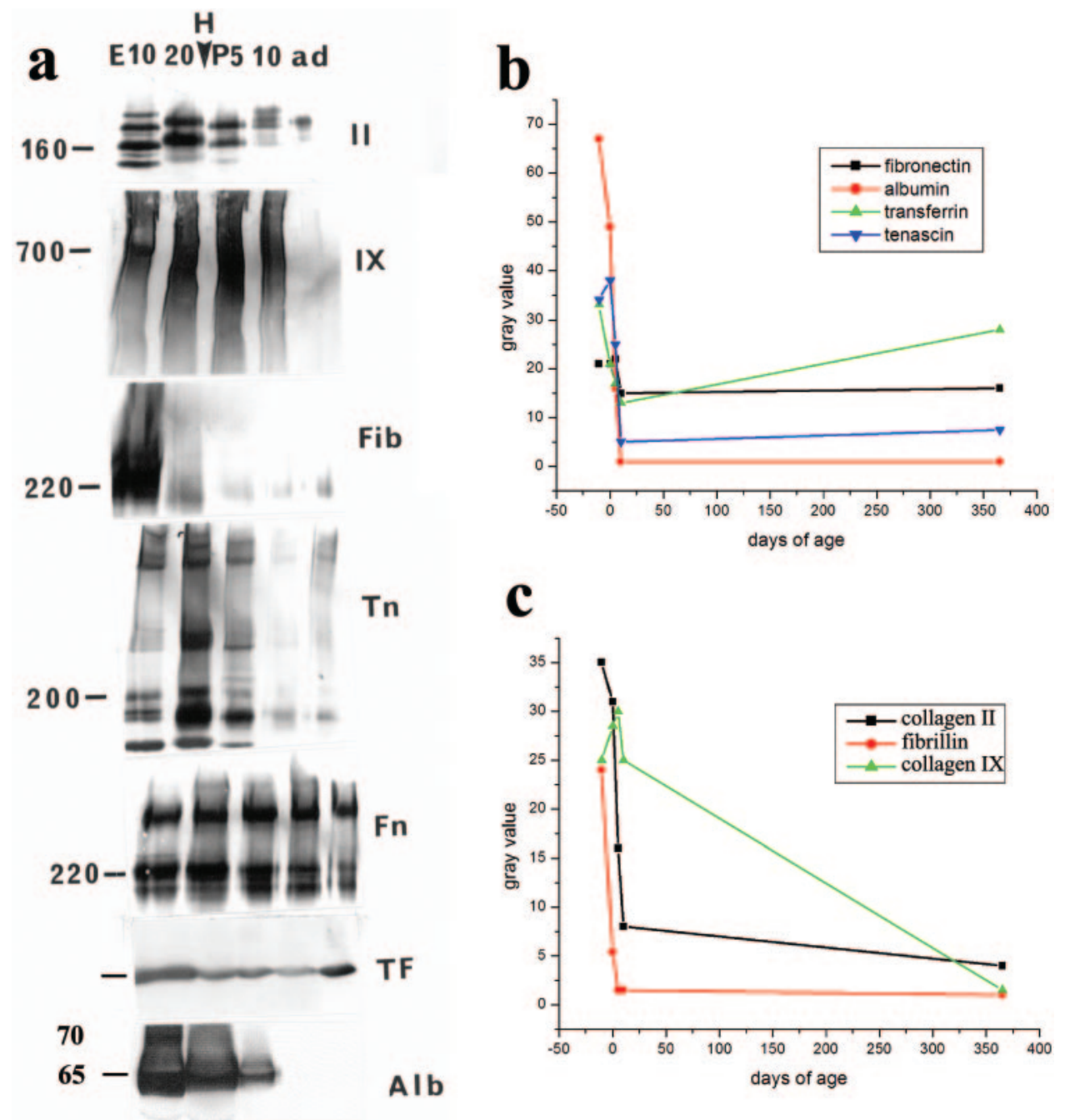

Figure 6. Western blot analysis (a) showing the abundance of collagens II (II) and IX (IX), fibrillin (Fib), tenascin (Tn), fibronectin (Fn), transferrin (TF), and albumin (Alb) on $\mathrm{E} 10, \mathrm{E} 20, \mathrm{P} 5$, and $\mathrm{P} 10$ and in the adult. $\mathrm{H}$, hatching at E21. In each lane, $40 \mu \mathrm{L}$ of $\mathrm{VB}$ was loaded. With the exception of fibronectin and transferrin, all proteins declined in concentration shortly after hatching. The protein bands from the Western blots were quantified by scanning densitometry $(\mathbf{b}, \mathbf{c})$. The gray values of the proteins shown in the keys were plotted against age of the chickens (days of age).

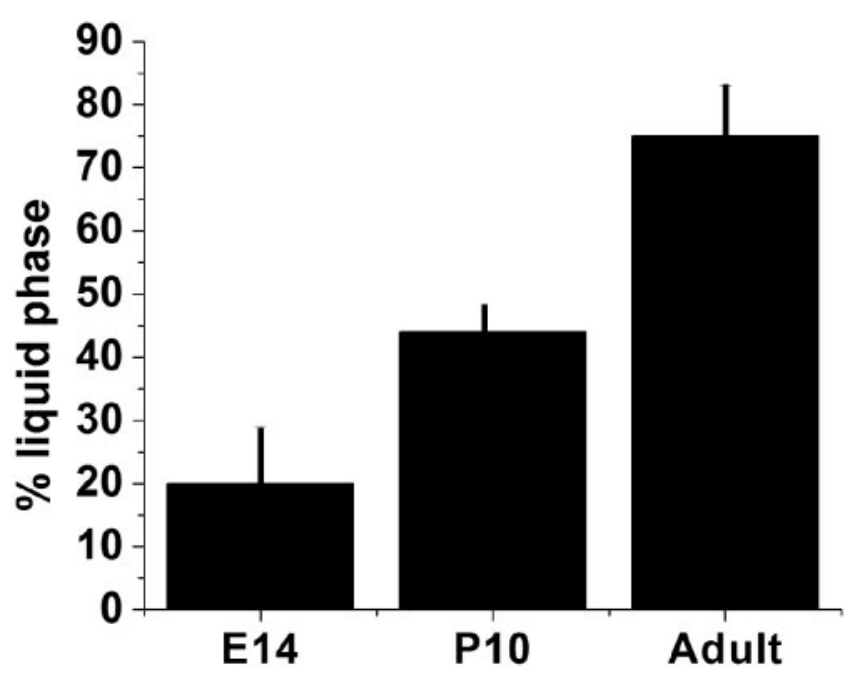

Figure 7. Liquefaction of the chick VB with increasing age. The percentage of liquid versus gel VB was determined in E14, P10, and adult chicken. The percentage of liquid VB increased from $20 \%$ at late embryogenesis to $>40 \%$ at $\mathrm{P} 10$ and $>70 \%$ after 1 year of age. $n=6$ at each stage.
mRNA in the retina shows that glial cells, which were traditionally considered to be the main source of ILM production, play only a minor role in ILM protein synthesis. Our assumption is in agreement with studies in the mouse showing that the ILM protein mRNAs are abundant in lens and ciliary body but absent in the retina. ${ }^{27,28}$ The hybridization studies in mice were performed using radioactively labeled cRNA probes, which have a higher sensitivity than the DIG-labeled probes used in this study. Further support for the origin of ILM proteins from lens and ciliary body and not from retina comes from grafting experiments. Mouse retinal transplants grafted into chick eyes were covered with an ILM that was derived from the chick host and not from the mouse retinal transplant. ${ }^{23}$ Yet, we cannot entirely exclude the possibility that retinal glial cells also contribute to the synthesis of the ILM. Disruption in the ILM and/or pial BM in mouse mutants shows that the main function of retinal or cortical glia is to provide the cellular receptors necessary for BM assembly. ${ }^{7,13}$

The synthesis of ILM proteins by mainly extraretinal tissues implies that for the assembly of new or the turnover of already existing ILM, its ECM constituents have to present in the VB. The highest concentration of ILM proteins in VB is expected to be present during early embryogenesis when the retina expands by $25 \%$ per day and large segments of new ILM have to be assembled. As shown in Figures 3 and 4, the highest concentrations of ILM proteins were indeed detected during that time. Maximum ILM protein concentrations were detected 

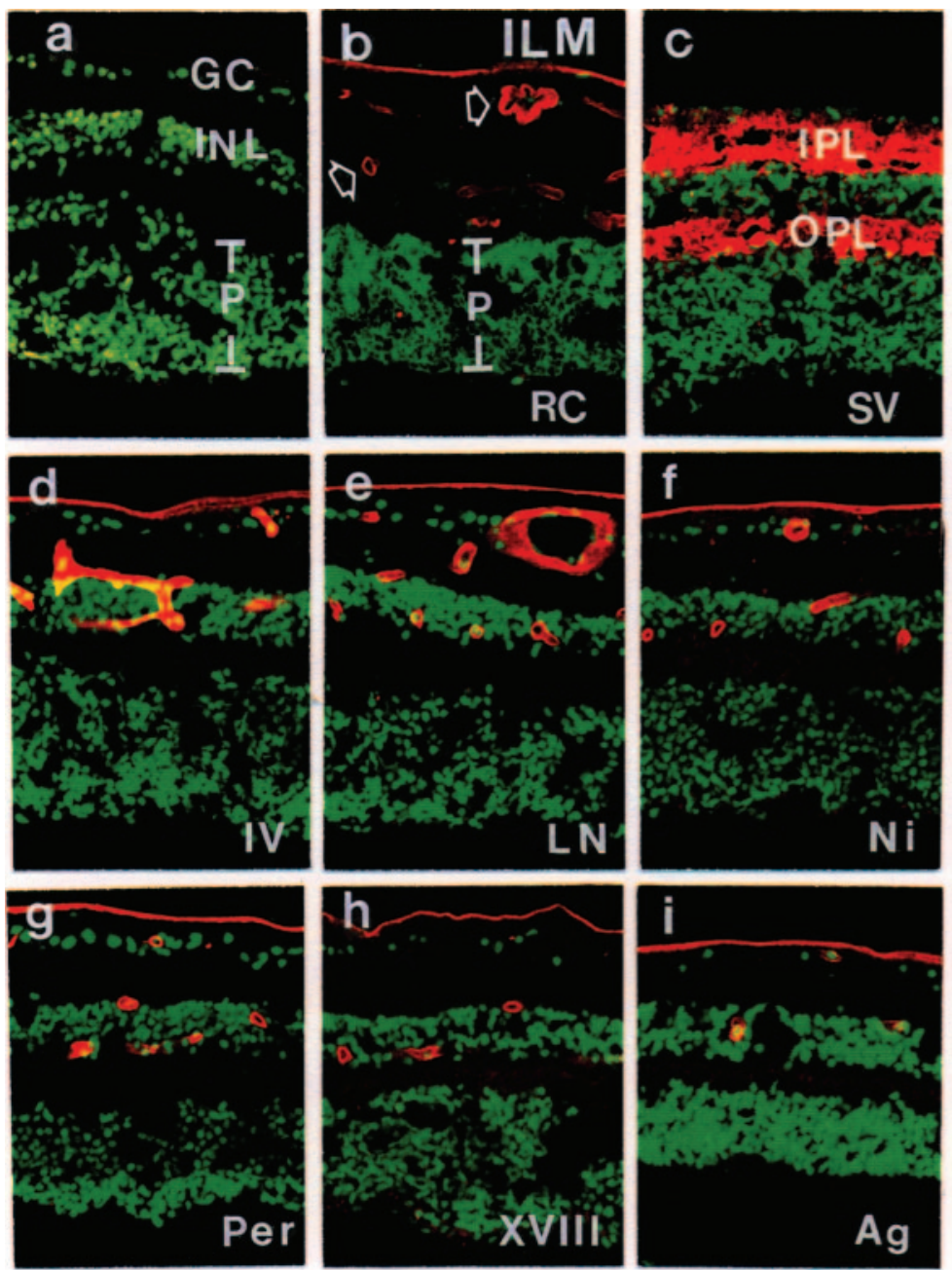
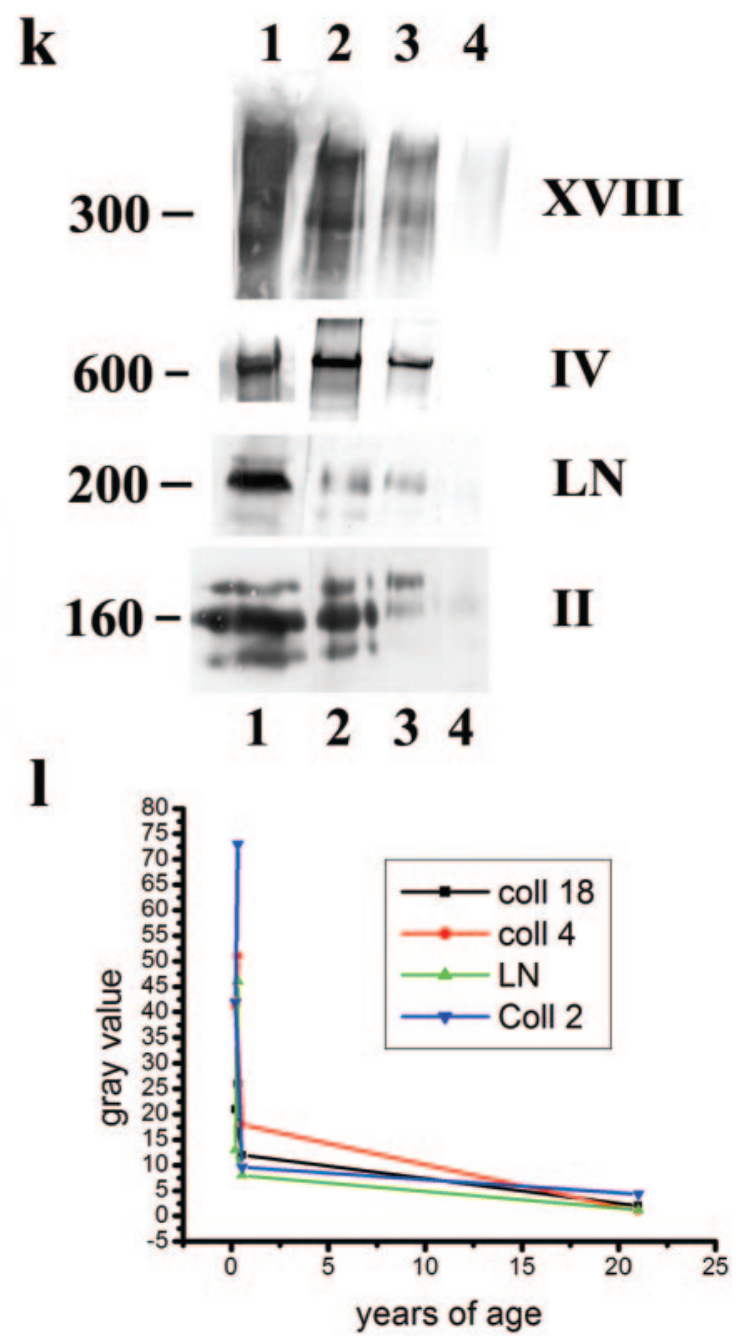

FiguRE 8. Cross sections of adult human retina stained for ILM protein constituents (d-i). For orientation, sections were also stained with a generic nuclear marker (green in $\mathbf{a}, \mathbf{c}, \mathbf{d}-\mathbf{i}$ ), with the RC2 marker for photoreceptors (P; green in $\mathbf{b}$ ), and the SV2 marker for the inner (IPL) and outer (OPL) plexiform layers (c, red). The section in (b) was also stained for laminin to show the location of the retinal vasculature (red; arrows) in the ganglion cell (GC), the IPL and the inner nuclear layer (INL). Collagen IV (IV), laminin-1 (LN), nidogen (Ni), perlecan (Per), collagen XVIII (XVIII), and agrin (Ag) were detected in the ILM and the BMs of the retinal blood vessels (d-i, red). Western blot analysis (k) showed the presence of laminin-1, collagen XVIII, collagen IV, and collagen II in human VB. The VB samples were pooled from three 19-week-old fetal eyes (lane 1), a 2.4-month (lane 2) and 6.3-month-old (lane 3) eyes, and from an adult eye (lane 4). Densitometry (1) showed that the proteins were most abundant in fetal VB, less abundant at 2.5 months after birth, even less at 6.5 months of age, and almost undetectable in the adult VB. Bar, $50 \mu \mathrm{m}$.

between E10 and E20, at which time the retina reaches its maximum surface area, and no new ILM has to be assembled. Shortly after reaching peak concentrations, the ILM protein concentrations decreased precipitously. Even more surprising, the concentrations of the ILM proteins in the adult VB were at their lowest levels, strongly suggesting that ILM turnover throughout adult life is minute. Immunocytochemistry of adult chick and human retina showed that all BM proteins were present in the ILM, indicating that ILM proteins in the ILM are well protected from degradation. Consistent with the rapid decrease of protein after hatching in chicks, the mRNA expression for ILM proteins is already downregulated during embryogenesis, preceding the decrease of protein by at least 10 days.

Data similar to those in chicks were obtained for the synthesis of the ILM in human eyes, as we found that the highest $\mathrm{BM}$ protein concentrations in VB were found in fetal eyes. Shortly after birth and 6 months further in development, ILM protein synthesis was greatly reduced, and the proteins were barely detectable in the adult VB.
It has to be noted that in addition to laminin-1, other laminin family members are synthesized in the eye. They originate from retinal cells and provide an ECM in the photograph receptor layer, but are not present in the ILM. ${ }^{31}$

There are, to our knowledge, no previous publications regarding the metabolism of the ILM. However, studies in other systems show that BMs are long-lived tissue structures. In frog, for example, freezing of muscle fibers leads to the death of the muscle cells but leaves the BM sheaths intact. $^{32}$ Further, crushing of nerves in the peripheral nervous system leads to the elimination of myelin, whereas the BMs of the Schwann cells survive and serve as conduits for the regenerating axons. ${ }^{33}$ Finally, radiolabeling ECM proteins in rat showed that the glomerular BMs in the kidney have turnover rates greater than 100 days, ${ }^{34}$ much slower than that of other proteins of the kidney. Our current data are consistent with these studies, as they show that ILM proteins have a very slow turnover rate and survive for very long times. 


\section{Metabolism of the VB}

Our study also established a developmental regulation of VB synthesis that is similar in its time course to that of the ILM. VB proteins are very abundant in embryogenesis, greatly downregulated after hatching, and present only at very low levels in the adult. Particularly striking is the very early downregulation of fibrillin between E10 and E20 (Fig. 6, Fib). The only ECM protein that is present in VB at similar levels throughout life is fibronectin (Fig. 6, Fn). As shown by in situ hybridization (Fig. $2 \mathrm{e}$ ), fibronectin is not synthesized in the eye, but rather originates from extraocular tissues, and it is currently unclear how it gets into the VB. Albumin and transferrin were also detected in chick VB. While albumin is downregulated shortly after hatching, transferrin stays in VB at high levels throughout life. Transferrin most likely originates from the ciliary body, ${ }^{30}$ and we assume the same is true for albumin. Obviously, not all the proteins secreted by the ciliary epithelium undergo developmental regulation. In human eyes, collagen II appears to be similarly regulated as in chick, with high concentration in embryogenesis, and very low concentration in the adult eye. The decrease in collagen II synthesis in the human eye occurs within 2 years after birth.

There are earlier reports on the turnover of the VB ECM proteins. Some laboratories have found an age-related decrease of collagen II in VB and postulated that de novo synthesis of collagen II and other VB proteins does not occur in the adult eye. ${ }^{35}$ Quantification of collagen II and IX mRNAs in mouse eyes by Northern blot analysis showed that both collagen mRNAs have their highest expression during embryogenesis, followed by a sharp decline shortly after birth. Barely detectable levels of collagen and IX mRNA were found in the adult eye. ${ }^{36}$ The time course very much resembles the downregulation of VB proteins in the chick and human, as presented herein. Investigators in other laboratories have found that the concentration of collagen II remains steady and have postulated that some de novo synthesis of collagens occurs in the adult. 37,38

A series of findings in human eyes are consistent with a low level of VB synthesis in the adult. Age-related liquefaction (synchysis), the aggregations of collagen fibers (syneresis, "floaters"), and the posterior detachment of the VB (PVD) from the ILM in $30 \%$ of humans older than 60 years ${ }^{15-17}$ are signs of gradual degradation and low synthesis of VB in the adult. ${ }^{39,40}$ In addition, the VB does not regenerate after vitrectomy. Finally, a recent study in humans detected a continuous decline of collagen IX concentration in human VB, with a half-life of collagen IX of approximately 11 years. ${ }^{41}$ All data strongly suggest that the VB, similar to the ILM, is metabolized very little and undergoes a slow degradation with age. Liquefaction of the VB is well established in humans and starts with the first 5 years of life. We showed that VB liquefaction also occurs in chick, with an early onset after hatching.

The question arises as to why ILM and VB synthesis is restricted to embryonic stages of development and why ILM and VB synthesis decreases so dramatically after birth or hatching. We speculate that ILM and VB are particularly essential during embryonic development but are dispensable later in life. Experimental removal of the ILM at early embryonic stages leads to a series of abnormalities, such as retinal dysplasia, retinal ectopias and a massive loss of ganglion cells. ${ }^{4-6}$ ILM removal later in development does not cause these abnormalities, ${ }^{4}$ and ILM peeling and vitrectomy in adult humans is in fact the method of choice in macular hole surgery.

It is well established that other ECM structures in the body are very slowly metabolized as well. Articular cartilage and tendon, for example, have a very slow turnover and are considered nonregenerative tissue structures. ${ }^{42}$ We speculate that, for the maintenance of ECM structures, evolution favored slow turnover over a continuous and rapid metabolism. Collagens and proteoglycans are inherently protected from degradation by their helical structure or their GAG side chains, and a constant high synthesis of ECM proteins carries the risk of excessive ECM buildup, as seen in fibrosis and BM thickening in diabetes. ${ }^{43,44}$ We speculate that many ECM structures, including the ILM and VB, are assembled during embryogenesis and then are maintained at a very low level of turnover.

\section{Acknowledgments}

The authors thank Robert Johnson from the Brain and Tissue Bank for Developmental Disorders (University of Maryland) for excellent assistance in providing us with the human tissue samples, and Jack Greenwalt for the adult chicken.

\section{References}

1. Yurchenco PD. Basal lamina assembly. Curr Opin Cell Biol. 1994; 6:674-681

2. Timpl R, Brown JC. Supramolecular assembly of basement membranes. Bioassays. 1996;18:123-132.

3. Erickson AC, Couchman JR. Still more complexity in mammalian basement membranes. J Histochem Cytochem. 2000;48:12911306.

4. Halfter W. Disruption of the retinal basal lamina during early embryonic development leads to a retraction of vitreal endfeet, and increased number of ganglion cells, and aberrant axon outgrowth. J Comp Neurol. 1998;397:89-104.

5. Halfter W, Dong S, Balasubramahni M, Bier M. Temporary disruption of the retinal basal lamina and its effect on retinal histogenesis. Dev Biol. 2001;238:79-96.

6. Halfter W, Willem M, Mayer U. Basement membrane-dependent survival of retinal ganglion cells. Invest Ophthalmol Vis Sci. 2005; 46:1000-1009.

7. Georges-Labouesse E. Essential role of $\alpha 6$ integrins in cortical and retinal lamination. Curr Biol. 1998;8:983-986.

8. Nakano I, Funahashi M, Takada K, Toda T. Are breaches in the glia limitans the primary cause of the micropolygyria in Fukujama-type congenital muscular dystrophy (FCMD)? - pathological study of the cerebral cortex of an FCDM fetus. Acta Neuropatbol. 1996; 91:313-321.

9. Williams RS, Swisher CN, Jennings M, Ambler M, Caviness VS. Cerebro-ocular dysgenesis (Walker-Warburg syndrome): neuropathologic and etiologic analysis. Neurology. 1984;34:1531-1541.

10. Gleeson JG, Walsh CA. Neuronal migration disorders: from genetic diseases to developmental mechanisms. Trends Neurosci. 2000; 23:352-359.

11. Graus-Porta D, Blaess S, Senften M, et al. Beta1-class integrins regulate the development of laminae and folia in the cerebral and cerebellar cortex. Neuron. 2001;16:367-379.

12. Halfter W, Dong S, Yip YP, Willem M, Mayer U. A critical role of the pial basement membrane in cortical histogenesis. $J$ Neurosci. 2002;22:6029-6040.

13. Moore SA, Saito F, Chen J, et al. Deletion of brain dystroglycan recapitulates aspects of congenital muscular dystrophy. Nature. 2002; 418:422- 425.

14. Costell M, Gustafsson E, Aszodi A, et al. Perlecan maintains the integrity of cartilage and some basement membranes. $J$ Cell Biol. 1999;147:1109-1122.

15. Sebag J. The Vitreous: Structure, Function and Patbobiology. New York: Springer Verlag; 1989.

16. Bishop PN. Structural macromolecules and supramolecular organization of the vitreous gel. Prog Retin Eye Res. 2000;19:323-344.

17. Ihanamaeki T, Pelliniemi L, Vuorio E. Collagens and collagenrelated matrix components in the human and mouse eye. Prog Retin Eye Res. 2004;23:403-434.

18. Allen WS, Ottenbein EC, Wardi AH. Isolation and characterization of the sulfated glycosaminoglycans of the human vitreous body. Biochem Biophys Acta. 1977;498:167-175. 
19. Yada T, Suzuki S, Kobayashi K, et al. Occurrence in chick embryo vitreous humor of a type IX collagen proteoglycan with an extraordinary large chondroitin sulfate chain and a short a1 polypeptide. J Biol Chem. 1990;265:6992-6999.

20. Bishop PN, McLeod D, Reardon A. Effects of hyaluronan lyase, hyaluronidase, and chondroitin ABC lyase on mammalian vitreous gel. Invest Ophthalmol Vis Sci. 1999;40:2173-2178.

21. Bishop PN, Takanosu M, le Goff M, Mayne R. The role of the posterior ciliary body in the biosynthesis of vitreous humor. Eye. 2002; 16:454-460.

22. Ring C, Hassell J, Halfter W. Expression pattern of collagen IX and potential role in segmentation of the peripheral nervous system. Dev Biol. 1996;180:41-53.

23. Halfter W, Dong S, Schurer B, et al. Protein composition, synthesis and assembly of the embryonic chick retinal basal lamina. Dev Biol. 2000;220:111-128.

24. Dong S, Landfair J, Balasubramahni M, Bier M, Cole G, Halfter W. Expression of basal lamina protein mRNAs in the early embryonic chick eye. J Comp Neurol. 2001;447:261-273.

25. Linsenmayer TF, Gibney E, Gordon M, Marchant JK, Hayashi M, Finch JM. Extracellular matrices of the developing chick retina and cornea: localization of mRNAs for collagen II and IX by in situ hybridization. Invest Opbthalmol Vis Sci. 1990;31:1271-1276.

26. Perez R, Halfter $W$. Tenascin protein and mRNA in the avian visual system: distribution and potential contribution to retinotectal development. Perspect Dev Neurobiol. 1994;2:75-87.

27. Sarthy PV, Fu M. Localization of laminin B1 mRNA in retinal ganglion cells by in situ hybridization. J Cell Biol. 1990;110:2099-2108.

28. Dong L-J, Chung AE. The expression of the gene for entactin, laminin A, laminin B1 and laminin B2 in murine lens morphogenesis and eye development. Differentiation. 1991;48:157-172.

29. Halfter W, Dong S, Schurer B, Cole GJ. Collagen XVIII is a heparan sulfate proteoglycan. J Biol Chem. 1998;273:25404-25412.

30. Bertazolli-Filho R, Laicine EM, Haddad A. Synthesis and secretion of transferrin by isolated ciliary epithelium of rabbit. Biochem Biophys Res Comm. 2003;305:820-825.

31. Libby RT, Champliaud M-F, Claudepierre T, et al. Laminin expression in adult and developing retinae: evidence of two novel CNS laminins. J Neurosci. 2000;20:6517-6528.
32. Sanes JR, Mashall LM, McMahan UJ. Reinnervation of muscle fiber basal lamina after removal of myofibers: differentiation of regenerating axons at original synaptic sites. J Cell Biol. 1978;78:176198.

33. Ide C, Tohyama K, Yokota R, Nitatori T, Onodera S. Schwann cell basal lamina and nerve regeneration. Brain Res. 1983;288: 61-75.

34. Price RG, Spiro RG. Studies on the metabolism of the renal glomerular basement membrane. J Biol Chem. 1977;252:85978602.

35. Balazs EA, Denlinger JL. Aging changes the vitreous. In: Sehuler R, Kline D, Dismukes K, eds. Aging and Human Visual Function. New York: Alan R. Liss, Inc.; 1982:45-57.

36. Ihanamaeki T, Salminen H, Saeaemanen A-M, et al. Age-dependent changes in the expression of matrix components in the mouse eye. Exp Eye Res. 2001;72:423-431.

37. Swann DA, Constable IJ. Vitreous structure. I. Distribution of hyaluronate and protein. Invest Opbthalmol Vis Sci. 1972;11:159163.

38. Bishop PN, Reardon A, McLeod D, Ayad S. Identification of alternatively spliced variants of type II procollagen in vitreous. Biochem Biophys Res Commun. 1994;203:289-295.

39. Sebak J. Age-related changes in human vitreous structure. Graefes Arch Clin Exp Ophthalmol. 1987;225:89-93.

40. Los LI, von der Worp RJ, van Luyn MJA, Hooymans JMM. Agerelated liquefaction of the human vitreous body: LM and TEM evaluation of the role of proteoglycans and collagen. Invest $O p b$ thalmol Vis Sci. 2003;44:2828-2833.

41. Bishop PN, Holmes DF, Kadler KE, McLeod D, Bos KJ. Age-related changes on the surface of vitreous collagen fibrils. Invest Opbthalmol Vis Sci. 2004;45:1041-1046.

42. Hamerman D. The biology of osteoarthritis. N Engl J Med. 1989; 320:1322-1330.

43. Ljubimov AV, Burgeson RE, Butkowski RJ, et al. Basement membrane abnormalities in human eyes with diabetic retinopathy. J Histochem Cytochem. 1996;44:1469-1479.

44. Tsilibary EC. Microvascular basement membranes in diabetes mellitus. J Pathol. 2003;200:537-546. 